\title{
Perfil de niños con déficits en la cognición numérica*
}

\section{Profile of Children with Deficits in Numerical Cognition}

\author{
Recepción: 03 Abril 2016 | Aprobación: 07 Febrero 2017
}

\author{
INDIRA ARIAs-RoDRIGUEZ \\ Universidad Estadual Paulista, Brasil \\ Jéssica-Mendes Nascimento \\ Universidad Estadual Paulista, Brasil \\ Flávia-Heloísa SAntos ${ }^{\mathrm{a}}$ \\ Universidad Estadual Paulista, Brasil \\ ORCID: http://orcid.org/0000-0003-2592-9038
}

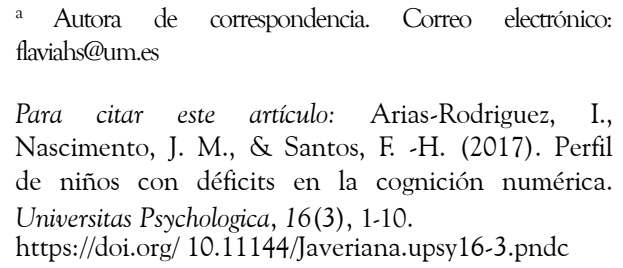

\section{RESUMEN}

La presente investigación tuvo como objetivo caracterizar el perfil neurocognitivo de niños en relación con las habilidades cuantitativas, intelectuales, de memoria operativa y sus aspectos emocionales. La muestra fue de 42 niños de edad escolar de 8 a 10 años, divididos en dos grupos: con dificultades en aritmética $(\mathrm{CDA}=21)$ y $\sin$ dificultades en aritmética $(\mathrm{SDA}=21)$, que fueron evaluados a través de pruebas cognitivas y escalas de comportamiento, que analizaron variables como: rendimiento escolar, razonamiento abstracto, memoria operativa, cognición numérica, ansiedad y estrés. El perfil de los niños CDA se caracterizó por dificultades en el rendimiento escolar de forma general. Se encontraron síntomas de estrés compatibles con fases de alerta y resistencia, baja capacidad de memoria operativa y de cognición numérica, específicamente de procesamiento numérico y cálculo.

\section{Palabras clave}

aritméticas; discalculia; cognición numérica; estrés; memoria operativa

\begin{abstract}
This study aimed to characterize the neurocognitive profile of children regarding quantitative, intellectual, and mnemonic skills; and their emotional state. The sample consisted of 42 school children 8-10 years old, divided into two groups: those with difficulties in arithmetic (CDA $=21)$ and without difficulties in arithmetic (SDA $=21)$. The children were evaluated through cognitive tests and behaviour scales, which analysed variables such as scholar achievement, abstract reasoning, working memory, numerical cognition, anxiety and stress. The profile of CDA children was characterized by global difficulties in schooling achievement. Also they had stress symptoms compatible with alert and resistance phases, low capacity in working memory and numerical cognition, specifically for numerical processing and calculation.

Keywords

arithmetic; dyscalculia; numerical cognition; stress; working memory
\end{abstract}




\section{Introducción}

Cohen Kadosh y Walsh (2009) definieron cognición numérica como 'los procesos de activación cerebral que implican la semántica y el significado numérico'. Por ejemplo, la cantidad "cinco" corresponde a un dígito (5), números romanos (V), estímulos no simbólicos (••• • $\bullet)$, etc. La cognición numérica está influenciada por factores biológicos, cognitivos, educacionales y culturales (Santos, Silva, Ribeiro, Dellatolas, \& von Aster, 2012) y posee cuatro sistemas: 1) Sentido Numérico; 2) Producción numérica; 3) Comprensión numérica y 4) Cálculo (Silva, Ribeiro, \& Santos, 2015).

El Sentido numérico es la capacidad de reconocer, comparar, sumar y restar cantidades pequeñas sin la necesidad de contar (Dehaene, 1997). En la medida en que se adquieren experiencias cotidianas y académicas con cantidades, se va formando un sistema métrico interno que se orienta espacialmente y ordena tales cantidades de forma continua, como de cero a diez, mil, infinito...

McCloskey, Caramazza y Basili (1985) indicaron que el procesamiento numérico implica tanto la comprensión numérica, es decir, la comprensión de la naturaleza de los símbolos numéricos y sus cantidades, como la producción numérica, que se refiere a la escritura, la lectura y el conteo de números u objetos. En cuanto al componente relacionado con el cálculo, los autores lo dividen en dos subsistemas: cálculo mental y cálculo escrito. Ambos requieren la capacidad de entender los signos matemáticos y el acceso a los datos aritméticos básicos, como sumas elementares, tablas de multiplicación y algoritmos de dominio para operaciones básicas.

Von Aster y Shalev (2007) presentaron un modelo de cognición numérica, caracterizado por procesos cognitivos que determinan el desarrollo de las habilidades cuantitativas y numéricas concomitantes a la progresión académica. Se trata de cuatro sistemas: 1) cardinal: es un proceso innato y automático para la determinación de la magnitud de un pequeño conjunto de ítems, proporcionando una comprensión básica de los números; 2) verbal: es representación lingüística de cantidades; los niños aprenden a asociar un número de objetos o eventos a palabras oídas y/o escritas; 3) visual arábigo: constituye el desarrollo de la simbolización numérica en el que las cantidades pasan a ser asociadas con los dígitos en forma arábiga; 4) ordinal: la línea numérica mental se desarrolla en función de la experiencia con cantidades y corresponde a una imagen mental de los números cuya capacidad aumenta gradualmente a lo largo de los años, así como, paralelamente, se desarrolla la capacidad de memoria operativa.

Silva y Santos (2011) investigaron los aspectos de la cognición numérica y la memoria operativa en niños escolares. Los participantes con déficit en aritmética exhibieron puntuaciones ligeramente más bajas que los niños sin perjuicios en el nivel intelectual, así como déficits específicos en la memoria operativa visuoespacial y déficits en procesamiento numérico y cálculo. Ribeiro y Santos (2017) utilizando un protocolo similar, también encontraron estas características que son compatibles con el diagnóstico de Discalculia del Desarrollo (DD).

La DD, o Trastorno Específico del Cálculo (Organización Mundial de Salud [OMS], 1993), se revela como una dificultad para realizar las operaciones básicas de adición, substracción, multiplicación y división, sin que ello sea causado por una educación inadecuada o deficiencia intelectual (Kaufmann \& von Aster, 2012). En pruebas estandarizadas como Zareki-R, el diagnóstico de DD se puede establecer por 1.5 desviación estándar por debajo del promedio, tanto en el perjuicio en tres subtest como en la puntuación total del instrumento (Rotzer et al., 2009).

El Consenso Internacional denominó DD primaria a los déficits atribuidos exclusivamente a disfunciones de la cognición numérica (Kaufmann et al., 2013) que caracteriza por dificultades aritméticas graves y persistentes (Kaufmann \& von Aster, 2012). En contraste, se clasifica como DD secundaria a los niños con déficits en la cognición numérica asociados a i) disfunciones cognitivas no numéricas, por ejemplo memoria operativa o ii) otros 
trastornos del desarrollo psicológico comórbidos, como la dislexia (Kaufmann et al., 2013). Los déficits en la cognición numérica de niños con $\mathrm{DD}$ en general son persistentes a pesar de las intervenciones convencionales (Ribeiro \& Santos, 2017).

Algunos niños con discalculia adquieren una actitud negativa hacia el estudio de matemáticas, haciendo aún más difícil la adquisición de las competencias cuantitativas básicas; en muchos casos. se evidencia una ansiedad a las matemáticas en particular (Rubinsten \& Tannock, 2010) y la presencia de síntomas depresivos (Cruvinel \& Boruchovitch, 2004).

El objetivo de este estudio fue caracterizar el perfil de niños con déficits en la aritmética, teniendo en cuenta el rendimiento escolar, habilidades cognitivas y aspectos del comportamiento, haciendo una comparación con niños con desarrollo típico a través de la evaluación neurocognitiva.

\section{Método}

\section{Participantes}

Fueron seleccionados para el estudio 42 niños, 18 chicos y 24 chicas de 8 a 10 años de edad. Los niños fueron divididos en dos grupos: con dificultades aritméticas (CDA, N $=21) \mathrm{y}$ sin dificultades aritméticas (SDA, N $=21$ ), tomando como criterios generales de exclusión los niños diagnosticados con trastornos neurológicos, psiquiátricos, desnutrición o con uso de sustancias psicoactivas.

Para el grupo CDA los criterios de inclusión fueron: dificultades relacionadas con las matemáticas indicadas por el profesor del niño, confirmadas por la nota de corte en el test de aritmética, nota de corte $<9$ (en el caso de los niños de tercer grado) o $<14$ (en el caso de los niños de cuarto grado), previstas en el manual del Test de Rendimiento Escolar (Stein, 1994), como resultado inferior al esperado para el grado cursado, sin embargo, con razonamiento abstracto esperado para su edad cronológica. Fueron seleccionados para el grupo
SDA niños con ausencia de reprobaciones de año escolar, de trastornos de aprendizaje, así como quejas de dificultades de aprendizaje. Todos los participantes fueron estudiados a través de una cuidadosa evaluación neurocognitiva. Los datos se especifican en la Tabla 1.

\section{TABLA 1}

Aspectos sociodemográficos de la muestra

\begin{tabular}{llll}
\hline Edad en Años & 8 & 9 & Total \\
\hline$N(\%)$ & $16(45.24)$ & $26(61.9)$ & $42(100)$ \\
Sexo (\%) chicos & $7(16.67)$ & $11(26.19)$ & $18(42.86)$ \\
Sexo (\%) chicas & $9(21.4)$ & $15(35.71)$ & $24(57.14)$ \\
\hline$N(\%)$ distribución de los años escolares de acuerdo con la edad & \\
\hline $3 .^{\circ}$ & $10(23.81)$ & - & $10(23.81)$ \\
$4^{\circ}$ & $6(14.29)$ & $26(61.9)$ & $32(76.19)$ \\
\hline
\end{tabular}

Fuente: elaboración propia.

\section{Procedimientos}

El estudio fue aprobado por el Comité de Ética de la UNESP, proceso N. - 31415114.2.1001.5398, con fecha 23 de septiembre del 2014 y los padres de los niños firmaron un término de consentimiento libre. Las pruebas se llevaron a cabo en orden semialeatorio (intercalando tareas verbales y no verbales); las tareas de lápiz y papel fueron realizadas primero que las tareas computarizadas. Se aplicaron en tres sesiones diferentes y duración total media de 120 minutos, con intervalos para descanso, utilizando siempre el mismo orden de exposición para todos los niños.

\section{Materiales}

Anamnesis: adaptada del cuestionario elaborado por Santos (2002). Matrices Progresivas coloridas de Raven-MPCR (Raven, 1992). Adaptación brasileña (Angelini, Alves, Custódio, Duarte, \& Duarte, 1999). Test de Rendimiento Escolar (Stein, 1994). Batería de Test Neuropsicológicos de Procesamiento Numérico y Cálculo para niños, versión revisada, Zareki-R (von Aster \& Dellatolas, 2006). Adaptación brasileña y datos normativos presentados por Santos, Silva, Ribeiro, Dellatolas et al., 2012 \& Santos, Silva, 
Ribeiro, Dias et al., 2012. Automated Working Memory Assessment ([AWMA], Alloway, 2007). Para una descripción de los test, consulte Ribeiro y Santos (2012); la adaptación brasileña está descrita en Santos y Engel (2008). Inventario de Síntomas de Estrés Infantil ([ISS-I], Lipp \& Lucarelli, 1998). Escala de Ansiedad a las Matemáticas ([EAM], Carmo \& Figueiredo, 2005).

\section{Resultados}

\section{Anamnesis}

Las historias clínicas evidencian una muestra homogénea en factores relacionados con el nacimiento de los niños, el uso del portugués como un único idioma hablado en casa, la edad de los padres y la situación socioeconómica de las familias.

Los grupos también fueron similares con respecto a los antecedentes gestacionales de la madre. Para cada grupo se presentaron dos casos de hipertensión, 1 amenaza de aborto y 1 hemorragia, lo que representa $19.05 \%$ de incidencia para cada grupo. No se encontraron diferencias significativas entre los grupos para los variables parto y peso al nacer.

Con respecto al parto, se presentaron nueve casos de nacimientos prematuros en el grupo CDA, que representan $28.6 \%$ de la muestra y tres en el grupo SDA, lo que equivale a 14.3 $\%(p=0.27)$. Referente al peso al nacer, se reportaron cinco casos de bebés que nacieron con peso entre $1500 \mathrm{~g}$ y $2500 \mathrm{~g}$ en el grupo CDA, lo que representa el $22.7 \%$ y solo un $8.3 \%$ en el grupo SDA $(p=0.22)$. Los niños que poseían bajo peso al nacer no mostraron evidencias de daño cerebral.

En relación con el desarrollo neuropsicomotor, los niños CDA demoraron más tiempo en meses para el control de esfínteres, control cervical y para gatear, según recuerdan sus padres. Presentando diferencias significativas entre los grupos, constatado estadísticamente con efecto de gran magnitud $(\mathrm{d}>0.9)$, para el control cervical $(t=3.08 ; p=0.04 * ; d=0.98)$ y el control del tronco $(t=2.04 ; p=0.04 * ; d=2.9)$.

\section{Raciocinio abstracto}

El análisis de los percentiles reveló similitud entre los grupos CDA y SDA para la edad en meses respecto al raciocinio abstracto: $F(1,40)$ $=1.97 ; p=0.17 ; \eta^{2}=0.005 ;$ Percentil: $F(1$, $40)=2.73 ; p=0.11 ; \eta^{2}=0.07$, los grupos se clasificaron como III + (Intelectualmente promedio Superior) (Tabla 2).

\section{Rendimiento escolar}

Todos los niños del grupo CDA tuvieron rendimiento inferior al esperado para el año escolar cursado (Stein, 1994) en subtest de escritura, aritmética y la puntuación total, asî como $76.19 \%$ de la muestra con dificultades en la lectura, resultado opuesto al grupo SDA donde todos los niños estaban por encima de las expectativas para el año escolar en todas los subtest y puntaje bruto total (Tabla 2).

En cuanto a los niños CDA, $57.14 \%$ de la muestra obtuvo puntuaciones abajo de lo esperado, referentes al año anterior cursado en el subtest de aritmética: 2 niños de tercer grado y 10 de cuarto; en el subtest de escritura fue encontrado 1 caso de tercer grado y 8 de cuarto, correspondiente al $42.86 \%$ y para el subtest de lectura, rendimiento inferior al esperado para el año anterior fue percibido en cuatro casos, lo que representa $19.05 \%$ del total; para la puntuación bruta total 7 niños de cuarto grado mostraron resultados inferiores a los datos normativos para el año anterior, lo que equivale al $33.33 \%$ de la muestra (Stein, 1994).

Se encontraron diferencias significativas para todos los subtest del Test de Rendimiento Escolar en los análisis entre los grupos CDA y SDA: Escritura $\left[F(1 ; 33.52)=31 ; p<0.0001 ; \eta^{2}\right.$ $=0.44]$, Aritmética $[F(1,40)=31.001 ; p<$ $\left.0.0001 ; \eta^{2}=0.46\right]$, Lectura $[F(1 ; 32.86)=14.2$; $\left.p=0.001 ; \eta^{2}=0.26\right]$ y el resultado total $[F(1$, 40) $\left.=38.8 ; p<0.0001 ; \eta^{2}=0.49\right]$. 


\section{Cognición numérica}

La Tabla 2 presenta los resultados [media (desviación estándar)] obtenido por los niños CDA y SDA de 3.․ y 4..$^{\circ}$ grado, en la evaluación de la batería neuropsicológica de procesamiento numérico y cálculo Zareki-R. Los niños CDA alcanzaron puntuaciones más bajas en comparación con los niños SDA en todos los subtest de la batería.

Sentido numérico: No hubo efecto de grupo para la enumeración de puntos $[F(1,40)=$ $\left.2.71 ; p=0.11 ; \eta^{2}=0.06\right]$, sin embargo, para la estimación visual se hallaron diferencias significativas entre los grupos $[F(1,40)=6.22 ; p$ $\left.=0.02 ; \eta^{2}=0.13\right]$.

Producción numérica: Para los tres subtest de Zareki-R que involucran esta capacidad, el grupo CDA obtuvo peores puntuaciones en comparación con el grupo SDA y en todos los casos estas diferencias resultaron significativas. Conteo oral en orden inverso: $[F(1 ; 25.30)$ $\left.=24.85 ; p<0.001 ; \eta^{2}=0.38\right]$, dictado de números: $\left[F(1 ; 23.36)=25.33 ; p<0.001 ; \eta^{2}=\right.$ 0.39]; lectura de números: $[F(1,40)=15.45$; $p$ $\left.<0.001 ; \eta^{2}=0.28\right]$.

Comprensión numérica: Para los tres subtest de Zareki-R que implican esta capacidad, el grupo CDA presentó peores puntuaciones en comparación con los niños del grupo SDA. Comparación oral: $\left[F(1 ; 40)=5.72 ; p=0.02 ; \eta^{2}\right.$ $=0.13]$, comparación escrita: $[F(1 ; 40)=0.76$; $\left.p=0.39 ; \eta^{2}=0.02\right]$, estimativa contextual $[F(1$; 40) $\left.=1.42 ; p=0.24 ; \eta^{2}=0.03\right]$.

Cálculo: Para los subtest Cálculo Mental y Problemas Aritméticos de la Zareki-R, el rendimiento de los niños SDA fue superior al de los niños CDA. Estos resultados fueron corroborados por el "tamaño del efecto", observándose que la mayoría de las magnitudes de efecto estuvo arriba de 0.6. Cálculo mental $\left[F(1 ; 29.11)=59.48 ; p<0.001 ; \eta^{2}=0.29\right] \mathrm{y}$ Problemas aritméticos $[F(1 ; 30.08)=68.03 ; p$ $\left.<0.001 ; \eta^{2}=0.63\right]$. Para la puntuación total Zareki-R hubo diferencias significativas, los niños CDA presentaron puntuaciones más bajas que los niños SDA $\left[F(1 ; 40)=86.93 ; p<0.001 ; \eta^{2}\right.$ $=0.68]$.

\section{TABLA 2}

Puntuaciones [media (desviación estándar)] obtenidas por los grupos CDA y SDA para el raciocinio abstracto, Test de Rendimiento Escolar y ZAREKI-R

\begin{tabular}{lllll}
\hline & $\begin{array}{l}\text { Tercer Grado } \\
\text { CDA(N=5) }\end{array}$ & SDA(N=5) & $\begin{array}{l}\text { Cuarto Grado } \\
\text { CDA(N=16) }\end{array}$ & SDA(N=16) \\
\hline Razonamiento abstracto & $22.8(4.6)$ & $24(3.74)$ & $25.19(3.82)$ & $27.31(4.83)$ \\
\hline Rendimiento escolar & & & & \\
\hline PB. Escritura & $13.8(2.28)$ & $24(2.24)$ & $19.25(6.06)$ & $27.06(3.75)$ \\
PB. Aritmética & $6.8(1.3)$ & $10.8(0.45)$ & $10.75(2.46)$ & $17.13(2.63)$ \\
PB. Lectura & $58.6(6.11)$ & $64.2(5.59)$ & $63.31(4.76)$ & $68.31(1.14)$ \\
PB. Total & $79(7.28)$ & $99(7.35)$ & $93.31(9.94)$ & $112(6.16)$ \\
\hline Cognición numérica & & & & \\
\hline Sentido numérico & $9.2(1.64)$ & $10.2(0.45)$ & $8.69(2.73)$ & $11.07(2.67)$ \\
\hline Enumeración de puntos & $3.6(0.55)$ & $3.4(0.89)$ & $3.19(0.5)$ & $3.69(0.48)$ \\
Estimación visual & $5.6(1.67)$ & $6.8(1.1)$ & $5.25(2.52)$ & $7.38(2.5)$ \\
\hline Producción numérica & $18.8(8.7)$ & $34.8(0.84)$ & $28.69(5.87)$ & $35.1(1.29)$ \\
\hline Conteo inverso & $1.2(0.84)$ & $3.6(0.55)$ & $2.44(1.31)$ & $3.69(0.48)$ \\
Dictado den números & $8(4)$ & $15.2(0.84)$ & $12.38(2.68)$ & $15.38(1.09)$ \\
Lectura de números & $9.6(4.34)$ & $16(0)$ & $13.88(2.87)$ & $16(0)$ \\
\hline Comprensión numérica & $40.8(3.03)$ & $42(7.87)$ & $41(4.12)$ & $46.1(4.96)$ \\
\hline Comparación oral & $14.8(1.1)$ & $14(1.41)$ & $12.75(2.7)$ & $14.94(1.12)$ \\
Comparación escrita & $18.8(1.79)$ & $17.2(6.26)$ & $18.25(2.82)$ & $19.75(0.68)$ \\
Estimación contextual & $7.2(3.35)$ & $10.8(3.03)$ & $10.63(3.56)$ & $11.38(4.36)$ \\
\hline Cálculo & $5.8(3.7)$ & $44.4(2.97)$ & $23.01(9.83)$ & $44.1(5.68)$ \\
\hline Cálculo mental & $4(2.35)$ & $34.8(2.95)$ & $18.19(9.03)$ & $33.25(5.42)$ \\
Problemas aritméticos & $1.8(1.79)$ & $9.6(1.14)$ & $4.81(3.22)$ & $10.88(1.71)$ \\
\hline Total & $87.8(15.83)$ & $153.4(10.41)$ & $116.5(17.49)$ & $158.44(10.56)$ \\
\hline
\end{tabular}

Notas: CDA: con dificultades aritméticas, SDA: sin dificultades aritméticas. Razonamiento abstracto: puntuaciones obtenidas en el Test MPC. ZAREKI-R: Batería de Test Neuropsicológicos de Procesamiento Numérico y Cálculo para niños. PB: Puntuación Bruta. Fuente: elaboración propia.

\section{Memoria operativa}

Se observaron diferencias significativas entre los grupos para todos los subtest $(\mathrm{p}<0.05)$ y por lo tanto se encontraron diferencias significativas para las cuatro categorías conceptuales: memoria a corto plazo verbal $[F(1,39)=38.62 ; p$ $\left.<0.001 ; \eta^{2}=0.5\right]$, memoria a corto plazo visuoespacial $\left[F(1,39)=74.93 ; p<0.001 ; \eta^{2}\right.$ $=0.66]$, memoria operativa verbal $[F(1,31.57)$ $\left.=21.33 ; p<0.001 ; \eta^{2}=0.35\right]$ y visuoespacial $\left[F(1,39)=57.68 ; p<0.001 ; \eta^{2}=0.6\right]$, en los que el grupo CDA obtuvo peor rendimiento que el grupo SDA (Tabla 3). 


\section{TABLA 3}

Puntuaciones [Media (Desviación Estándar)] obtenidas por los grupos CDA y SDA en el Test AWMA

\begin{tabular}{lllll}
\hline AWMA & $\begin{array}{l}\text { Tercer Grado } \\
\text { CDA }(N=5)\end{array}$ & SDA $(N=5)$ & $\begin{array}{l}\text { Cuarto Grado } \\
\text { CDA( } N=16)\end{array}$ & SDA(N=16) \\
\hline Almacenamiento & & & & \\
\hline Memorización de digitos & $20.2(3.56)$ & $24.2(3.42)$ & $21.56(3.77)$ & $27(3.54)$ \\
Recordar palabras & $17.4(2.51)$ & $20.38(2.55)$ & $23.4(2.30)$ & $22.13(2.36)$ \\
Recordar pseudopalabras & $9(3.08)$ & $11.56(2.48)$ & $15.6(3.85)$ & $16.53(4.26)$ \\
& & & & \\
\hline MCPV & $46.6(3.05)$ & $56.14(2.82)$ & $60.56(3.31)$ & $65.66(3.39)$ \\
\hline Matriz de puntos & $15(2.92)$ & $13.69(2.21)$ & $20(2.45)$ & $22.87(3.6)$ \\
Memoria para laberintos & $11.64(4.67)$ & $13.63(4.4)$ & $17.20(3.9)$ & $21.2(3.32)$ \\
Recordación de Bloques & $14.4(3.72)$ & $16.31(3.4)$ & $20.2(4.6)$ & $21.13(4.19)$ \\
\hline MCPVE & $41(3.77)$ & $43.62(3.34)$ & $57.4(3.65)$ & $65.2(3.7)$ \\
\hline Procesamiento & & & & \\
\hline Juicio de frases & $7.2(2.59)$ & $8.56(2.25)$ & $10.4(2.07)$ & $10.47(2.53)$ \\
Recordación de conteos & $4.8(2.59)$ & $6.44(2.44)$ & $8.6(1.82)$ & $12.27(4.24)$ \\
Recordar digitos inversos & $6.8(4.44)$ & $8.88(2.63)$ & $8.8(2.95)$ & $12.07(3.56)$ \\
\hline MOV & $18.8(3.21)$ & $23.88(2.44)$ & $27.8(4.87)$ & $34.81(3.44)$ \\
\hline Discriminación de formas & $12.2(3.96)$ & $10.19(2.95)$ & $13.4(4.72)$ & $17.67(3.13)$ \\
Juicio espacial & $7.8(1.92)$ & $11.63(3.12)$ & $11.6(3.58)$ & $17.73(4.89)$ \\
Span Espacial & $6.6(4.51)$ & $12.63(3.93)$ & $14.4(0.89)$ & $17.8(4.46)$ \\
MOVE & $26.6(3.46)$ & $34.45(3.33)$ & $39.4(3.06)$ & $53.2(4.16)$ \\
\hline
\end{tabular}

Notas: CDA: con dificultades aritméticas, SDA: sin dificultades aritméticas. AWMA: Automated Working Memory Assessment. MCPV: Memoria a Corto Plazo Verbal. MCPVE: Memoria a Corto Plazo Visuoespacial. MOV: Memoria Operativa Verbal. MOVE: Memoria Operativa Visuoespacial. Fuente: elaboración propia.

\section{Escalas comportamentales}

Los niveles de ansiedad y estrés de las escalas indican diferencias significativas entre los grupos CDA y SDA, en las que los niños CDA reportaron más reacciones de estrés psicológico $\left[F(1,40)=4.58 ; p=0.04 ; \eta^{2}=0.1\right]$, reacciones psicológicas con componente depresivo $[F(1,40)$ $\left.=7.77 ; p=0.008 ; \eta^{2}=0.16\right]$, reacciones psicofisiológicas $\left[F(1,40)=7.48 ; p=0.009 ; \eta^{2}\right.$ $=0.16]$ y la suma de las reacciones de estrés $[F(1$, 40) $\left.=7.93 ; p=0.007 ; \eta^{2}=0.17\right]$. Hubo reportes de síntomas que indican estrés para el $66.7 \%$ de los niños CDA, con 7 niños en la fase de alerta y 7 en la fase de la resistencia y el $38.1 \%$ de los niños del grupo de SDA, con 7 niños en la primera fase y solo 1 en la fase 2. El análisis de la Escala de Ansiedad a las Matemáticas mostró un equilibrio entre los grupos $[F(1,40)=0.52 ; p=0.48$; $\left.\eta^{2}=0.01\right]$, presentando niveles moderados de ansiedad a la asignatura de matemáticas (Tabla 4).
TABLA 4

Puntuaciones [media (desviación estándar)] obtenidas por los grupos CDA y SDA en las Escalas de Comportamiento

\begin{tabular}{lllll}
\hline \multicolumn{3}{c}{ Tercer Grado } & Cuarto Grado \\
\hline & $\operatorname{CDA}(N=5)$ & SDA $(N=5)$ & CDA $(N=16)$ & SDA $(N=16)$ \\
\hline ESI & & & & \\
Reacciones físicas & $13(4.42)$ & $7.4(4.34)$ & $8.94(6.38)$ & $7.63(4.94)$ \\
Reacciones psicológicas & $17.2(4.15)$ & $11(10.98)$ & $14.81(7.33)$ & $10.75(5.98)$ \\
R.P.C.D & $11.4(3.58)$ & $4.2(3.56)$ & $9.63(8.52)$ & $4.69(5.29)$ \\
R.P.F & $8(4.95)$ & $6.6(3.58)$ & $12(4.53)$ & $7.19(5.02)$ \\
Total & $49.6(12.26)$ & $29.2(18.46)$ & $45.38(21.59)$ & $30.31(18.56)$ \\
\hline EAM & $73.6(18.17)$ & $68.8(29.41)$ & $73.38(17.66)$ & $63.94(22.48)$ \\
\hline
\end{tabular}

Leyenda: CDA: con dificultades aritméticas, SDA: sin dificultades aritméticas. ESI: Escala de Stress Infantil. R.P.C.D: Reacciones psicológicas con componentes depresivos. RPF: Reacciones Psicofisiológicas. EAM: Escala de Ansiedad a las Matemáticas. Fuente: elaboración propia.

\section{Discusión}

El objetivo de este estudio fue caracterizar el perfil de niños con déficits en aritmética (CDA), teniendo en cuenta aspectos de rendimiento escolar, habilidades cognitivas y síntomas de ansiedad y estrés, en comparación con niños con desarrollo típico (SDA).

De acuerdo con Haase, de Souza Costa, Rettore Micheli, Silva Oliveira y Wood (2011) la etiología de los déficits relativos a la cognición numérica es multifactorial. En el presente estudio los datos de anamnesis revelaron homogeneidad de la muestra en variables en la mayoría de los parámetros evaluados. Por otro lado, se encontraron diferencias entre los grupos para el desarrollo neuropsicomotor. Aunque los hallazgos se basan en el relato de los padres, parecen confirmar la relación entre desarrollo cognitivo y neuropsicomotor (Araújo, 2002).

En el análisis del razonamiento abstracto, no se hallaron diferencias relativas a la capacidad intelectual de los grupos. El CIE-10 (OMS, 1993) señala que los trastornos de aprendizaje no son derivados de déficits en la inteligencia. Por otro lado, los resultados discrepan de algunos estudios que reportaron las dificultades en aritmética asociadas a un CI ligeramente más bajo (Ribeiro, 2013; Shalev, Manor, Auerbach, \& GrossTsur, 1998; Santiuste-Bermejo \& Santiuste-Díaz, 2008). En el presente estudio, los perjuicios en 
cognición numérica no pueden ser justificados por un bajo nivel intelectual.

En relación con el Rendimiento escolar las puntuaciones en los subtest de escritura, aritmética y total en los niños CDA resultaron por debajo de lo esperado para la edad y el grado cursado para los estudiantes de tercero y cuarto grado, estos últimos obtuvieron puntuaciones inferiores también para la lectura (Stein, 1994), hallazgos semejantes a los obtenidos por Silva y Santos (2011) y Ribeiro y Santos (2017). En la presente muestra, las puntuaciones fueron representativas de un trastorno mixto del desarrollo del aprendizaje escolar, entre tanto, los instrumentos del protocolo de investigación, aunque sugestivos, son insuficientes para el diagnóstico de dislexia.

Según von Aster y Shalev (2007), el sentido numérico es un aspecto generalmente preservado en niños con DD secundaria. En este estudio, no fueron presentadas diferencias significativas entre los grupos en cuanto al sentido numérico, las puntuaciones fueron equivalentes en escolaridad (Santos, Silva, Ribeiro, Dellatolas et al., 2012) y edad (Santos, Silva, Ribeiro, Dias et al., 2012). Además, corroboran otras muestras de niños con déficits en cognición numérica (Ribeiro, 2013; Silva \& Santos, 2011; Silva et al., 2015).

Los niños CDA exhibieron perjuicios en producción numérica, caracterizados por dificultades en la ejecución de tareas de adición y substracción esencialmente visuales, en la comprensión de los símbolos numéricos y en su reproducción; confirmando estudios previos (Gaitán \& Rey-Anacona, 2013; Ribeiro, 2013; Ribeiro \& Santos, 2017; Silva \& Santos, 2011; Silva et al., 2015).

Los déficits presentados en cálculo corresponden con perjuicios graves, esto es, muy por debajo de lo esperado para la edad y año escolar (Santos, Silva, Ribeiro, Dias et al., 2012). Son substanciales para el diagnóstico de DD (Kaufmann \& von Aster, 2012; Kaufmann et al., 2013) y similares a los observados en investigaciones previas (Gaitán \& Rey-Anacona, 2013; Ribeiro, 2013; Ribeiro \& Santos, 2017; Silva \& Santos, 2011; Silva et al., 2015).
Las puntuaciones obtenidas del estudio de la Memoria operativa son inferiores a Ribeiro y Santos (2012), tal como se observa en niños con DD secundaria (Kaufmann et al., 2013). Los hallazgos son también congruentes con los resultados de Skagerlund y Träff (2016), cuyos resultados de investigación con niños entre 8 y 10 años de edad $(\mathrm{N}=133)$ apuntaron que funciones ejecutivas como la memoria operativa están íntimamente relacionados con la capacidad matemática.

En las escalas de comportamiento, hubo un alto nivel de tensión en el rendimiento escolar para todos los alumnos según los datos normativos (Lipp \& Lucarelli, 1998). Sin embargo, el relato de los niños con dificultades de aprendizaje en matemáticas fue más expresivo en las reacciones de estrés psicológico, reacciones psicológicas con componente depresivo, suma de las reacciones de estrés y reacciones psicofisiológicas. Es decir, las dificultades escolares pueden causar ansiedad (Gracia-Bafalluy \& Escolano-Pérez, 2014). Sin embargo, no es posible determinar si el estrés es causa o consecuencia de las dificultades de aprendizaje de la muestra.

No se presentaron diferencias entre los grupos para la escala de ansiedad a las matemáticas, aunque, la mayoría de los niños mostró un nivel de ansiedad moderada. Los índices de ansiedad fueron superiores a los encontrados en la muestra de Ribeiro (2013) y Ribeiro y Santos (2017), lo que sugiere que el contexto escolar en el cual están insertados los niños del presente estudio puede ser amenazador, pues niños SDA también se mostraron ansiosos en esta escala.

Una limitación de este estudio fue el número reducido de participantes, sin embargo, los niños que componen la muestra fueron seleccionados a través de una cuidadosa evaluación y las variables en estudio se analizaron con rigor.

\section{Conclusión}

Los resultados de la evaluación neurocognitiva de los niños CDA arrojaron déficits en la cognición numérica acompañados de 
disfunciones cognitivas no numéricas (debido a los perjuicios en memoria operativa), y además es probable la comorbilidad con el trastorno de lectura. Estas características sugieren el diagnóstico de DD secundaria (Kaufmann et al., 2013). También hubo síntomas de estrés en estos niños compatibles con fases de alerta y resistencia.

\section{Agradecimientos}

Beca PEC-PEG del CNPq, Brasil ( Programa de Estudantes-Convênio de Pós-Graduação do Conselho Nacional de Desenvolvimento Científico e Tecnológico do Brasil)

\section{Referencias}

Alloway, T. P. (2007). Automated Working Memory Assessment Manual. Londres: Pearson Education Limited.

Angelini, A. L., Alves, I. C. B., Custódio, E. M., Duarte, W. F., \& Duarte, J. L. M. (1999). Manual: Matrizes Progressivas Coloridas de Raven. São Paulo, SP: Centro Editor de Testes e Pesquisas em Psicologia.

Araújo, A. P. Q. C. (2002). Avaliação e manejo da criança com dificuldade escolar e distúrbio de atenção. Jornal de Pediatria, 78(1), 104-110. https://doi.org/

Carmo, J. S., \& Figueiredo, R. (2005). Aprendizagem, emoção e ansiedade matemática: indícios e vestígios de histórias de punição e fracasso no ensino da matemática. Revista Trilhas (UNAMA), 7(15), 85-93.

Cohen Kadosh, R., \& Walsh, V. (2009). Numerical representation in the parietal lobes: Abstract or not abstract? Behavioral and Brain Sciences, 32, 313-328. https://doi.org/https:// doi.org/10.1017/S0140525X09990938

Cruvinel, M., \& Boruchovitch, E. (2004). Sintomas depressivos, estratégias de aprendizagem e rendimento escolar de alunos do ensino fundamental. Psicologia em Estudo,
9(3), 369-378. https://doi.org/10.1590/ S1413-73722004000300005

Dehaene, S. (1997). The number sense: How the mind creates mathematics. Nueva York: Oxford University Press.

Gaitán Chipatecua, A., \& Rey-Anacona, C. A. (2013). Diferencias en funciones ejecutivas en escolares normales, con trastorno por déficit de atención e hiperactividad, trastorno del cálculo y condición comórbida. Avances en Psicología Latinoamericana, 31(1), 71-85. https://doi.org/10.12804/ revistas.urosario.edu.co/apl/a.2347

Gracia-Bafalluy, M., \& Escolano-Pérez, E. (2014). Aportaciones de la neurociencia al aprendizaje de las habilidades numéricas. Revista de Neurología, 58(2), 69-76. Recuperado de https://biologiaunimetro.files.wordpress. com/2013/07/aportaciones-de-la-neurocie ncia-al-aprendizaje-de-las-habilidades-nu mc3a9ricas.pdf

Haase, V. G., de Souza Costa, D., Rettore Micheli, L., Silva Oliveira, L. F., \& Wood, G. (2011). O estatuto nosológico da discalculia do desenvolvimento. En Transtornos de aprendizagem 2: Da análise laboratorial e da reabilitação clínica para as políticas públicas de prevenção pela via da educação (pp. 139-144). São Paulo, SP: Memnon.

Kaufmann, L., Mazzocco, M. M., Dowker, A., von Aster, M., Göbel, S. M., Grabner, R. H., \& Nuerk, H. C. (2013). Dyscalculia from a developmental and differential perspective. Frontiers in Psychology, 4. https://doi.org/10.3389/fpsyg.2013.00516

Kaufmann, L., \& von Aster, M. (2012). The diagnosis and management of dyscalculia. Deutsche Ärzteblatt Internacional, 109(45), 767. https:// doi.org/10.3238/arztebl.2012.0767

Lipp, M. E., \& Lucarelli, M. D. (1998). Escala de Stress Infantil-ESI. Manual. São Paulo: Casa do Psicólogo.

McCloskey, M., Caramazza, A., \& Basili, A. (1985). Cognitive mechanisms in 
number processing and calculation: Evidence from dyscalculia. Brain and Cognitive, 4, 171-196. https:// doi.org/10.1016/0278-2626(85)90069-7

Organização Mundial da Saúde (1993). Classificação de transtornos mentais e de comportamento da CID-10: Descrições clínicas e diretrizes diagnósticas. Porto Alegre, RS: Artes Médicas.

Raven, J. C. (1992). Teste de Matrices Progresivas: Escala especial. Buenos Aires: Paidós.

Ribeiro, F. S. (2013). O efeito do treino musical sobre a capacidade da memória operacional e da cognição numérica de crianças com Discalculia do Desenvolvimento (Tesis de maestría inédita). Universidade Estadual Paulista. Recuperado de http://hdl.handle. net/11449/97434

Ribeiro, F. S., \& Santos, F. H. (2012). Treino musical e capacidade da memória operacional em crianças iniciantes, veteranas e sem conhecimentos musicais. Psicologia Reflexão e Critíca, 25, 559-567. https://doi.org/10.1590/ S0102-79722012000300016

Ribeiro, F. S., \& Santos, F. H. (2017). Enhancement of numeric cognition in children with low achievement in mathematic after a noninstrumental musical training. Research in Developmental Disabilities, 62, 26-39. https://doi.org/10.1016/j.ridd.2016.11.008

Rotzer, S., Loenneker, T., Kucian, K., Martin, E., Klaver, P., \& von Aster, M. (2009). Dysfunctional neural network of spatial working memory contributes to developmental dyscalculia. Neuropsychologia, 47, 2859-2865. https:// doi.org/10.1016/j.neuropsychologia. 2009.06.009

Rubinsten, O., \& Tannock, R. (2010). Mathematics anxiety in children with developmental dyscalculia. Behavioral and Brain Functions, 6(1), 46. https:// doi.org/10.1186/1744-9081-6-46

Santos, F. H. (2002). Memória operacional de crianças normais e com lesões congênitas: Desenvolvimento cognitivo e reorganização cerebral. São Paulo, Brazil: Universidade Federal de São Paulo-Escola Paulista de Medicina

Santos, F. H., \& Engel, P. M. J. (2008). Adaptação brasileira da AWMA, Automated Working Memory Assessment. En K. Z. Ortiz, L. I. C. Mendonça, A. Foz, C. B. Santos, D. Fuentes \& D. A. Azambuja (Orgs.), Avaliação neuropsicológica: Panorama interdisciplinar dos estudos na normatização e validação de instrumentos no Brasil (pp. 355-378). São Paulo: Vetor.

Santos, F. H., Silva, P. A., Ribeiro, F. S., Dellatolas, G., \& von Aster, M. (2012). Development of numerical representation among Brazilian schoolaged children. Jornal Internacional de Estudos em Educação Matemática, 5, 44-64. https://doi.org/10.5209/rev SJOP.2012.v15.n2.38862

Santos, F. H., Silva, P. A., Ribeiro, F. S., Dias, A. L. R.P., Frigério, M. C., Dellatolas, G., \& von Aster, M. (2012). Number processing and calculation in Brazilian children aged 7-12 years. Spanish Journal of Psychology, 15, 513-525. https://doi.org/10.5209/ rev_SJOP.2012.v15.n2.38862

Santiuste-Bermejo, V., \& Santiuste-Díaz, M. (2008). Consistencia epistémica del síndrome de dificultades del aprendizaje: aportaciones de la magnetoencefalografía como técnica de neuroimagen funcional. Universitas Psychologica, 7(3), 655-672. Recuperado de http://revistas.javeriana.ed u.co/index.php/revPsycho/article/view/384

Shalev, R. S., Manor, O., Auerbach, J., \& Gross-Tsur, V. (1998). Persistence of developmental dyscalculia: What counts? Results from a three year prospective follow-up study. Journal of Pediatrics, 133, 358-362. https://doi.org/10.1016/ S0022-3476(98) 70269-0

Silva, P. A., Ribeiro, F. S., \& Santos, F. H. (2015). Cognição numérica em crianças com transtornos específicos de aprendizagem. Temas em Psicologia, 23(1), 197-210. https:// doi.org/10.9788/TP2015.1-13 
Silva, P. A., \& Santos, F. H. (2011). Discalculia do desenvolvimento: Avaliação da representação numérica pela ZarekiR. Psicologia: Teoria e Pesquisa, 27, 169-177. https://doi.org/10.1590/ S0102-37722011000200003

Skagerlund, K., \& Träff, U. (2016). Processing of space, time, and number contributes to mathematical abilities above and beyond domain-general cognitive abilities. Journal of Experimental Child Psychology, 143, 85-101. https://doi.org/10.1016/ j.jecp.2015.10.016

Stein, L. M. (1994). TDE: Teste de Desempenho Escolar: Manual para aplicação e interpretação. São Paulo, SP: Casa do Psicólogo.

Von Aster, M. G., \& Dellatolas, G. (2006). ZAREKI-R: Batterie pour l'évaluation du traitement des nombres et du calcul chez l'enfant. Adaptation française [ZAREKIR: Batería para la evaluación del manejo numérico y de cálculo en el niño. Adaptación francesa]. París: Les Éditions du Centre de Psychologie Appliquée.

Von Aster, M. \& Shalev, R. S. (2007). Number development and developmental dyscalculia. Developmental Medicine $\mathbb{E}$ Child Neurology, 49(11), 868-873.

\section{Notas}

* Artículo de investigación. 